\title{
Building lasers inside cells
}

\section{Optical microresonators form stand- alone intracellular lasers.}

Light is a powerful tool in biology, and lasers are often used to excite fluorescent molecules for cellular fluorescence imaging. However, until recently, tiny lasers that could emit light from within cells were in the realm of science fiction, owing to technical challenges.

Seok Hyun Yun, of Harvard Medical School, and Matjaž Humar, a postdoc in his laboratory, overcame several obstacles to develop the first intracellular lasers. Early in his research, Yun, a physicist by training, noted that lasers "are big metal boxes ... not the form or material you can insert into the body," which limits their use in medicine to superficial regions of the body. He began to wonder why people had not used biomaterials to build lasers and speculated that small, biocompatible lasers could be used to study individual cell properties or be injected deep into tissues to deliver light to regions that are currently inaccessible.

Lasers need three parts to work: a pump source, a gain medium and an optical resonator. The pump source excites photons in the gain medium, which is often a fluorescent dye, and the optical resonator traps the photons, allowing them to amplify light by traveling back and forth in the gain medium to excite more photons. In principle, these components could be combined on scales small enough to build a laser inside a cell, but in practice it depends on identifying the right materials.

"As soon as I saw that fluorescent proteins and fluorescent dyes are very bright, I asked whether these might be good materials to build a laser," recalls Yun. Indeed, his group had already succeeded in using GFP as a gain medium in a cellular laser; but in that case, the optical resonator was outside of the cell. To build standalone intracellular lasers, they made use of fluorescent dyes. Finding an optical resonator that was small enough to fit inside a cell posed a greater challenge, as resonators are typically composed of mirrors that surround the gain medium.

Rather than trying to engineer microscopic mirrors, the team used an entirely different approach: whispering-gallery mode (WGM) resonators. WGM resonators are typically spherical and trap light as a result of internal reflection at the curved interface between materials of very different optical densities. The resonators can be composed of soft or hard materials with dimensions down to the submicrometer scale or even smaller.

The team developed intracellular lasers using three different microscale WGM resonators. The first was made up of oil droplets containing the fluorescent dye Nile red as the gain medium, which, when injected into cells, emitted laser light upon pulsed excitation. The team measured the force exerted by the cell on the droplets by making use of the fact that their emission spectra change when their shape deviates from a sphere.

The next resonators the team tested were naturally occurring lipid droplets in adipocytes. The researchers treated fat tissue from pigs with an enzyme to create round cells and added Nile red to form the gain medium. They excited the tissue using an optical fiber and found that adipocytes near the tip of the fiber exhibited lasing. In addition to these soft resonators, the team also used polystyrene beads as hard WGM resonators. The beads were readily taken up by cells and exhibited lasing when the gain medium dye was within the bead, on the surface of the bead or in the cytoplasm surrounding the bead.

Although the work was challenging, Yun attributes their ultimate success to time spent connecting the various building blocks of the project, including knowledge of biological systems and the physics of lasers. Intracellular lasers are still in their infancy, but their potential applica-

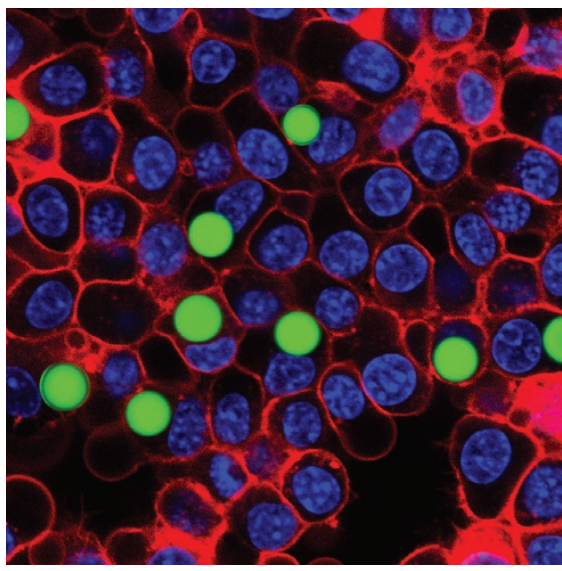

A field of cells containing lipid droplet microlasers (green) with labeled membranes (red) and nuclei (blue). Figure reproduced from Humar \& Yun, Nature Publishing Group.

tions are numerous. Given their narrow emission spectra and tunability based on resonator size and choice of gain medium, they could readily be used for multiplexed cell sorting.

In the long term, intracellular lasers could be used for deep-tissue photodynamic disease therapies. In such therapies, the intrinsic capabilities of cells, such as immune cells, to detect problems and travel to the disease site could be harnessed to target the lasers without human control. Further technical developments such as making the lasers smaller and brighter could facilitate an even wider range of uses. One could even imagine subcellular imaging of laser-labeled proteins built with nanoscale lasers targeted to proteins of interest. Given their broad potential, these intracellular lasers represent a major breakthrough in biotechnology.

\section{Rita Strack}

\section{RESEARCH PAPERS}

Humar, M. \& Yun, S.H. Intracellular microlasers. Nat. Photonics doi:10.1038/nphoton.2015.129 (27 July 2015). 